Original Paper http://ajol.info/index.php/ijbcs http://indexmedicus.afro.who.int

\title{
Intensification de la production agropastorale et genre dans le domaine soudano-sahelien au Burkina Faso
}

\author{
Katian NAPON ${ }^{1,2^{*}}$, Augustine AYANTUNDE ${ }^{2}$ et Dapola Evariste Constant DA ${ }^{1}$ \\ ${ }^{1}$ Université de Ouagadougou, Burkina Faso. \\ ${ }^{2}$ International Livestock Research Institute (ILRI) BP 1496 Ouagadougou, Burkina Faso. \\ *Auteur correspondant ; E-mail : katia.napon@gmail.com
}

\section{RESUME}

La faible pluviométrie et la pauvreté des sols du Séno et du Yatenga au Burkina Faso influencent négativement les rendements agricoles et la production animale. Pour améliorer la productivité et favoriser une meilleure sécurité alimentaire, il est nécessaire d'intensifier le système de culture à travers l'adoption de techniques culturales adéquates. L'objectif de cette étude est d'analyser la pratique des techniques d'intensification durable de la production agropastorale selon le genre. A cet effet, une enquête a été conduite auprès de 632 individus. Un modèle logistique binaire a été utilisé avec SPSS 20 pour vérifier si les caractéristiques sociodémographiques des enquêtés prédisaient de façon significative la pratique d'une technique d'intensification. Une valeur de $\mathrm{P}<0,05$ a été jugée statistiquement significative. Les résultats révèlent une différence en fonction du genre dans la pratique des techniques d'intensification de la production et montrent que ces différences sont liées au niveau d'éducation, à l'activité primaire, à la taille du ménage, au groupe ethnique, au groupe de genre et à l'âge des enquêtés. Les déterminants de l'adoption des technologies d'intensification de la production agropastorale devraient être pris en compte dans la politique agricole du pays pour une meilleure diffusion de ces technologies auprès des paysans.

(C) 2020 International Formulae Group. All rights reserved.

Mots clés : Technologies améliorées, systèmes mixtes, groupe de genre, Séno, Yatenga.

\section{Intensification of agro-pastoral production and gender in the Sudano-Sahelian domain in Burkina Faso}

\begin{abstract}
Low rainfall and poor soils in the Seno and Yatenga regions of Burkina Faso have a negative impact on agricultural yields and livestock production. To improve productivity and promote better food security, it is necessary to intensify the farming systems through the adoption of appropriate of intensification practices. The objective of this study is to analyze the effect of gender on intensification practices in agro-pastoral systems. A survey was conducted involving 632 individuals from different gender groups. A binary logistic model was used with SPSS 20 to assess whether the socio-demographic characteristics of the respondents had significant effect on intensification practices. A value of $\mathrm{P}<0.05$ was considered statistically significant. The results revealed
\end{abstract}


gender differences in intensification practices and showed that these differences are related to education level, primary activity, household size, ethnic group, gender group and age of the respondents. The determinants of the agro-pastoral production intensification technologies should be taken into account in the country's agricultural policy for a better dissemination of these technologies to farmers.

(C) 2020 International Formulae Group. All rights reserved.

Keywords: Improved technologies, mixed systems, gender group, Seno, Yatenga.

\section{INTRODUCTION}

La précarité des systèmes de production agricole dans le domaine Soudano-Sahélien compromet de plus en plus les besoins en nourritures des populations (Dorin, 2014; Camara et al., 2015). D'après le rapport du panel de Montpellier (2013), la croissance rapide de la population, des besoins alimentaires, la rareté des ressources naturelles (terre, eau et sols fertiles) couplées aux rendements agricoles stagnants et aux taux alarmants de famine et de malnutrition sont autant de facteurs aggravant la situation. La conception d'une agriculture plus productive et durable est un défi à relever pour assurer la sécurité alimentaire des populations et une exploitation durable des écosystèmes (Petit, 2011). La hausse de l'approvisionnement alimentaire des exploitations agricoles dépend d'une intensification agricole durable (Quisumbing et al., 2014). Les paysages agricoles d'Afrique de l'Ouest sont façonnés par les pratiques de gestion des agriculteurs, hommes et femmes. En outre, certains espaces et cultures agricoles spécifiques sont clairement liés au statut de femme ou d'homme (Howard et Nabanoga, 2007). Dans plusieurs cultures africaines, certains espaces agricoles sont classés linguistiquement comme « féminins » ou « masculins » (Alesina et al., 2011). Les problématiques sur l'accès et l'usage des ressources naturelles exposent les différences qui existent entre les sexes en la matière. Dans les secteurs des techniques agricoles, la même remarque est faite. Peterman et al. (2010) ont constaté dans la revue de 20 études de la dernière décennie sur les différences entre les sexes dans l'utilisation des intrants technologiques que dans seize de ces études, les hommes utilisaient plus d'intrants (engrais inorganique, insecticide, variétés de semences améliorées et puissance mécanique) que les femmes, comparativement aux quatre autres études où ce sont les femmes qui utilisaient davantage ces intrants (Croppenstedt et al., 2013).

Au Burkina Faso, l'agriculture est un secteur qui occupe plus de $80 \%$ de sa population (Herrera et Ilboudo, 2012), alors qu'elle est peu développée avec des techniques culturales encore à l'état embryonnaire. Il est crucial de bien expliquer les différences de genre qui existent dans l'adoption des stratégies d'intensification pour concevoir des politiques efficaces dans le double objectif de mettre fin aux écarts entre les hommes et les femmes et d'améliorer durablement de la productivité agricole (Theriault et al., 2017). Quelles variations selon le genre y'a-t-il dans le choix de pratiquer une technique d'intensification de la production agropastorale? Quelles sont les déterminants de la pratique d'une technique d'intensification durable?

Diverses études ont traité du genre dans l'agriculture et les systèmes d'intensification de la production agro-pastorale et de comment le genre peut influencer l'adoption d'une technologie (Djinadou et al., 2008 ; Hillenkamp, 2011 ; Parks et al., 2014 ; Smale et al., 2016 ; Theriault et al., 2017 ; Dabiré et al., 2018). Cependant, dans ces travaux le genre n'a pas été significativement associé à l'adoption des technologies et le sexe du chef de ménage est l'indicateur de genre utilisé. Par ailleurs, peu de travaux ont utilisé l'homme, la femme, et les jeunes comme l'indicateur de genre qui influence l'adoption des techniques d'intensification de la production agropastorale. C'est en cela que cette étude est innovatrice et trouve son intérêt en se proposant d'identifier les différentes techniques d'intensification pratiquées selon le genre et de chercher les déterminants de la pratique des techniques d'intensification durable de la production agro-pastorale par les populations. 


\section{MATERIEL ET METHODES \\ Sites de l'étude}

L'étude a été conduite dans les provinces du Séno et du Yatenga, situées respectivement au Nord et au Nord-ouest du Burkina Faso. (Figure 1). Ces deux provinces présentent des caractéristiques physiques relativement semblables. Elles appartiennent toutes deux au domaine sahélien avec des précipitations annuelles comprises entre 400 et $600 \mathrm{~mm}$ d'eau (pour le Séno), 500 et $700 \mathrm{~mm}$ (pour le Yatenga); et des sols relativement pauvres sous des formations végétales essentiellement constituées d'arbustes et d'épineux. L'agriculture et l'élevage sont les principales activités de la population. Il faut noter que dans la province du Séno, c'est l'élevage qui occupe principalement la population. Pour la campagne agricole 2017/2018, le Yatenga et le Séno ont produit respectivement 118964 tonnes et 73316 tonnes de céréales (INSD, 2018a, 2018b). L'orpaillage devient de plus en plus une activité non négligeable dans l'économie des ménages de ces provinces, particulièrement dans le Yatenga. Cette zone a d'importantes potentialités aurifères avec des exploitations artisanales de l'or disséminées un peu partout comme à Séguénéga, Guitti et Namissiguima (Capitant, 2017). Les conditions agroclimatiques difficiles de cette zone, font des technologies d'amélioration de la productivité une nécessité pour un essor de l'agropastoralisme.

\section{Echantillonnage et collecte des données}

La base de sondage utilisée est le Recensement Général de la Population et de l'Habitat de 2006 (RGPH). Dans chacune des deux provinces, quatre villages ont été concernés par l'étude. Il s'agit de Bani, Gorgadji, M'bamga et Seytenga pour le Séno et de Aorema, Bogoya, Tougou et Ziga pour le Yatenga. Un tirage aléatoire a permis de retenir 25 ménages par village, soit un total de 200 ménages concernés conformément aux objectifs de ce travail qui vise à analyser les choix, les pratiques et les rôles des hommes, des femmes, des jeunes garçons et des jeunes filles par rapport aux options d'intensification de la production agro-pastorale. L'approche mixte (quantitative et qualitative) a été la méthode utilisée. Une MARP (Méthode
Accélérée de Recherche Participative) réalisée au préalable sur les deux sites a permis d'identifier les pratiques agropastorales les plus courantes dans la zone. Ensuite, une enquête a été conduite auprès des ménages en mai 2017. Ainsi, dans chaque ménage, le chef, sa femme ou l'une de ses femmes, une jeune fille et un jeune garçon sont interviewés au moyen de questionnaires structurés. Le jeune ici désigne le garçon ou la fille, célibataire, exploitant un lopin de terre dans le ménage. Cependant, il faut noter que dans certains ménages il y'avait pas la catégorie des garçons et filles célibataires. De ce fait, au lieu d'un échantillon de 800 personnes, nous n'avons obtenu que 632 individus enquêtés.

\section{Gestion des données}

Dix pratiques d'intensification de la production agropastorale ont été considérées dans cette étude. Ce sont : l'usage de l'engrais et des pesticides, l'usage de la fumure animale, la plantation de variétés améliorées, les techniques de conservation des eaux et des sols (CES), le compostage, la mécanisation à petite échelle pour la production végétale ; l'élevage de races de bétail amélioré, l'usage de compléments alimentaire pour animaux, l'embouche, la collecte et la conservation du fourrage pour la production animale. Ces différentes techniques ont été mises en relation avec les caractéristiques sociodémographiques des personnes enquêtées. Le choix de ses pratiques s'explique par leur fréquence dans la zone d'étude. Par ailleurs, les variables sociodémographiques collectées ont été le groupe de genre, l'âge, le niveau d'éducation, l'activité principale, le statut matrimonial et le groupe ethnique.

\section{Analyses statistiques}

L'analyse des données a été effectuée à l'aide du logiciel IBM SPSS Statistics 20. Des analyses de fréquence et de moyennes ont été effectuées pour décrire les caractéristiques des personnes enquêtées et la pratique des techniques d'intensification. En général, l'analyse de l'influence de plusieurs prédicteurs sur une variable qualitative dépendante (avec deux catégories de réponses telles que "pratique" ou "non-pratique" d'une technologie) ne correspond pas à un modèle de 
probabilité linéaire en raison de ses faiblesses. Nous recherchons les déterminants de l'adoption des techniques d'intensification de la production agropastorale (ex: usage de l'engrais et des pesticides) dans les deux provinces. Le modèle logit est plus pratique ici car il donne un test de signification des paramètres permettant d'évaluer pour chaque variable retenue, le niveau de signification et son impact dans l'explication du phénomène étudié (Masuy-Stroobant et al., 2013). En outre, un avantage de l'utilisation d'un modèle logit est que les variables explicatives peuvent être un mélange de variables continues et qualitatives ou l'un des deux types (continu ou qualitatif). Nos données contiennent les deux types de variables et peuvent donc être utilisées correctement dans un modèle logit pour analyser les déterminants de l'adoption des technologies améliorées (Wooldridge, 2009). Un seuil de significativité minimum de $5 \%$ a été considéré pour l'interprétation des résultats.

\section{Modèle de régression logistique binaire :}

Dans la régression logistique binaire, le logarithme népérien du rapport de cotes est égal à une fonction linéaire des variables indépendantes exprimées en :

Logit $P i=\ln (P i / 1-P i)=\beta 0+\beta 1 X 1$

En utilisant la fonction anti $\log$ suivie d'une transformation mathématique, la valeur de Pi (probabilité de l'usage de l'engrais et des pesticides pour le ième répondant) est :

$P i=1 / 1+e^{-(\beta 0+\beta I X I)}$

Pour simplifier, l'équation (2) devient

$P i=1 / 1+e^{-Z I}$

où $\mathrm{ZI}=$ fonction des variables explicatives est exprimée comme suit :

$\mathrm{ZI}=\beta 0+\beta 1 X 1+\beta 2 X 2+\ldots \ldots+\beta n X n+u i(4)$ où : $\beta 0$ est la constante

$\beta 1 \ldots \beta n$ sont les coefficients de régression; par conséquent,

$Z i=\beta 0+\beta 1$ (Usage de l'engrais et des pesticides) $+\beta 2$ (Usage du fumier des animaux) $+\ldots \ldots+\beta 10$ (Mécanisation à petite échelle) (5)

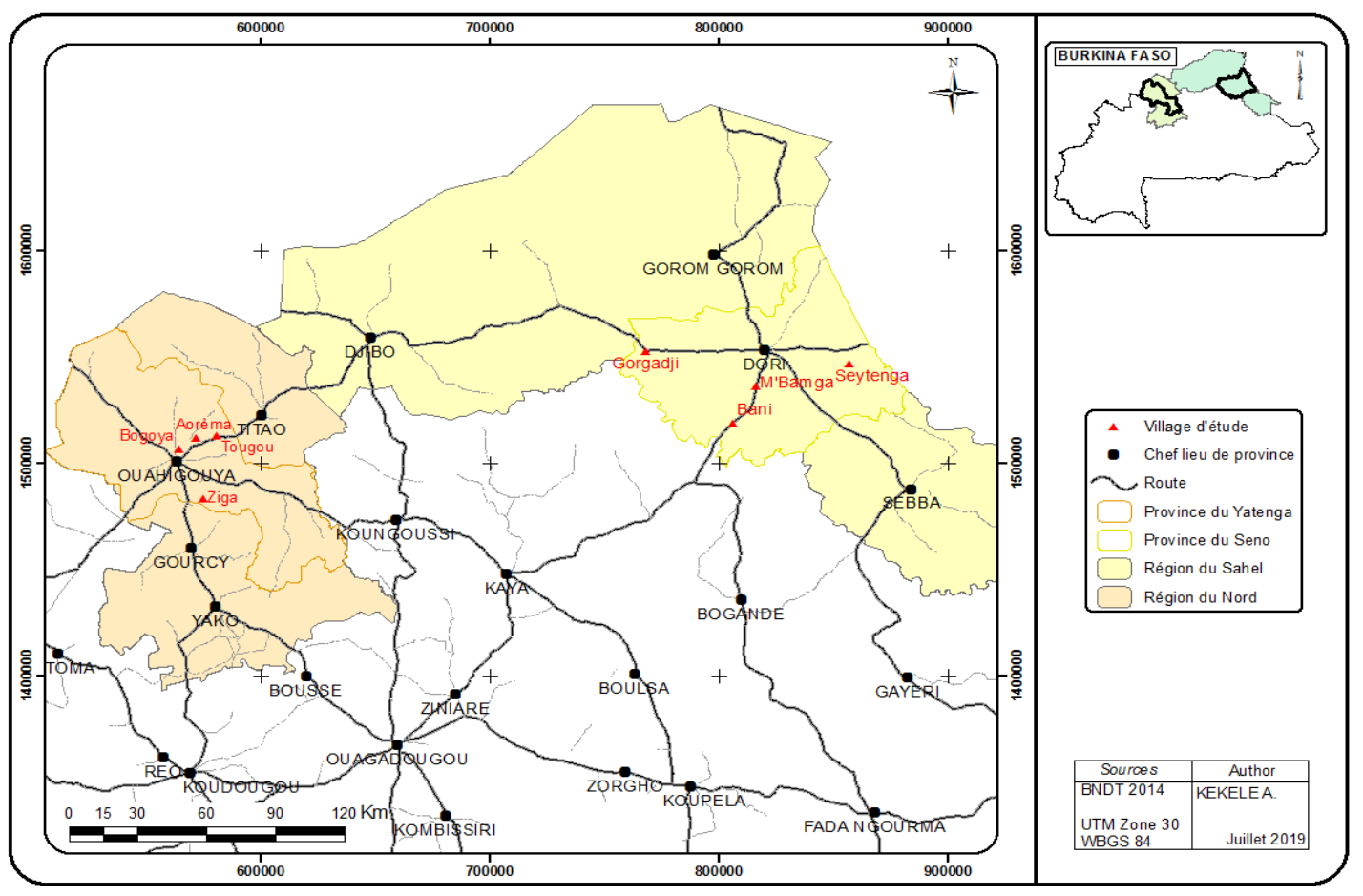

Figure 1 : Situation des sites de l'étude. 


\section{RESULTATS \\ Caractéristiques sociodémographiques des répondants dans les sites d'étude}

Selon les résultats de l'enquête, les groupes ethniques dominants sont les Mossis avec $99,7 \%$ des enquêtés du Yatenga et les peulhs, avec $80,2 \%$ des enquêtes dans le Séno que côtoie une minorité ethnique $(13,1 \%)$ constituée de Djermas, de Gourmantchés et de Haoussas (Tableau 1). Les principales activités sont l'agriculture et l'élevage. La combinaison agriculture et élevage concerne $72,4 \%$ des répondants dans le Séno contre $41,2 \%$ dans le Yatenga. La pratique exclusive de l'agriculture occupe $48,6 \%$ des répondants du Yatenga et $14,9 \%$ du Séno. Les niveaux d'instruction sont par ordre d'importance : la non-instruction, le primaire et le secondaire ou post secondaire. Le Séno $(60,4 \%)$ enregistre le plus fort taux de non instruits comparativement au Yatenga $(31,6 \%)$. Concernant la taille moyenne des ménages, elle est de dix-huit membres dans le Yatenga contre onze dans le Séno.

\section{Pratique des technologies d'intensification de la production selon le genre}

L'importance des pratiques
d'intensifications de la production agropastorale varie d'une province à l'autre. Les proportions d'hommes, de femmes, de jeunes garçons et de jeunes filles qui pratiquent les différentes technologies dans les deux zones sont présentées dans les Figures 2 et 3 . Dans la province du Séno, l'usage des engrais et des pesticides, le compostage pour l'intensification de la production végétale et l'élevage de races de bétail améliorées pour l'intensification de la production animale sont très faiblement pratiqués (Figure 2).

Seuls $3 \%$ des jeunes filles, $5 \%$ des hommes et des femmes élèvent des races améliorées de bétail. Egalement, 26\% des hommes, $22 \%$ des femmes, $19 \%$ des jeunes filles et $21 \%$ des jeunes garçons utilisent les engrais et les pesticides dans les champs. Le compostage lui est pratiqué par respectivement, $15 \%, 16 \%, 13 \%$ et $11 \%$, des hommes, des femmes, des jeunes filles et des jeunes garçons. A l'inverse, l'usage du fumier des animaux, l'utilisation de compléments alimentaires pour les animaux, l'embouche, la collecte et la conservation du fourrage sont les techniques d'intensification de la production agropastorale les plus pratiquées par les individus enquêtés (Figure 2). En effet, plus de 90\% des enquêtés hommes et femmes ainsi que les jeunes filles et garçons ont recours au fumier des animaux, aux compléments alimentaires, à la collecte et la conservation du fourrage pour les animaux et l'embouche comme techniques d'intensification de la production animale et végétale.

Dans la province du Yatenga (Figure 3), les techniques d'intensification de la production agropastorale considérées sont toutes très pratiquées par les répondants sans distinction de genre. Au moins $90 \%$ des hommes, femmes et jeunes utilisent le fumier des animaux, l'engrais et les pesticides, et font la collecte et la conservation du fourrage pour les animaux. Seul l'élevage de races de bétail améliorées semble peu pratiqué par les répondants avec moins de $50 \%$ des répondants femmes, jeunes filles et garçons contre 53\% des hommes qui adhèrent à cette technique. Globalement, la pratique des techniques d'intensification est plus forte chez les personnes enquêtées dans le Yatenga que dans le Séno. Au moins quatre sur cinq (4/5) des hommes, femmes ou jeunes garçons enquêtés dans le Yatenga pratiquent une technique d'intensification contre trois sur cinq $(3 / 5)$ pour le Séno.

Déterminants sociodémographiques de
l'utilisation des technologies d'intensification Les facteurs qui interviennent dans le choix de pratiquer une technique d'intensification de la production varient selon la technique et la province. En effet, l'analyse logistique a révélé que des caractéristiques sociodémographiques sont significativement associées à l'adoption des technologies d'intensification de la production. Dans la province du Séno, l'activité primaire, le niveau d'éducation, la taille du ménage, le groupe ethnique des individus enquêtés sont les facteurs qui se sont révélés significativement associés à 
la pratique des techniques d'intensification (Tableau 2).

L'utilisation des engrais et des pesticides $(\mathrm{p}=5 \%)$, des techniques de conservation des eaux et des sols et la mécanisation à petite échelle des activités agricoles $(\mathrm{p}=1 \%)$ sont des pratiques significativement associées à l'activité primaire des enquêtés. La plantation des variétés améliorées de céréales et de légumineuses et l'utilisation des techniques de conservation des eaux et des sols sont significativement associées au niveau d'instruction des enquêtés $(\mathrm{p}=1 \%)$. L'utilisation des engrais et des pesticides est aussi significativement associée à la taille du ménage ainsi qu'au groupe ethnique de l'enquêté $(\mathrm{p}=5 \%)$. Il y a une association significative $(\mathrm{p}=5 \%)$ entre l'utilisation des variétés améliorées et la taille du ménage. Il en est de même pour la collecte et la conservation du fourrage et le groupe ethnique de l'enquêté $(p=5 \%)$. Le groupe genre n'est pas significativement associé à la pratique des différentes techniques d'intensification de la production.

Dans la province du Yatenga, le niveau d'éducation, l'activité primaire, la taille du ménage, le groupe genre, l'âge des individus enquêtés sont les facteurs qui se sont révélés significativement associés à la pratique des techniques d'intensification (Tableau 3). L'utilisation des engrais et des pesticides, la collecte et la conservation du fourrage sont significativement associées à l'activité primaire $(\mathrm{p}=5 \%)$, de même que l'élevage de races améliorées de bétail $(\mathrm{p}=1 \%)$. La pratique de l'embouche et l'utilisation des variétés améliorées sont significativement associées au niveau d'instruction des enquêtés $(\mathrm{p}=5 \%)$. L'utilisation du fumier des animaux est associée à la taille du ménage. L'élevage des races améliorées est quant à lui significativement associé à la taille du ménage, au statut matrimonial et à l'âge de l'enquêté $(\mathrm{p}=5 \%)$. Cette dernière caractéristique est également significativement associée à l'utilisation des engrais et des pesticides, de même que le groupe de genre de l'enquêté.

Tableau 1 : Caractéristiques sociodémographiques de l'échantillon ( $\mathrm{n}=632)$.

\begin{tabular}{|c|c|c|c|}
\hline & & SÉNO (\%) & YATENGA (\%) \\
\hline \multirow{4}{*}{ Groupe de genre } & Homme & 36,6 & 26,9 \\
\hline & Femme & 36,9 & 27,2 \\
\hline & Jeune garcon & 14,6 & 25,0 \\
\hline & Jeune fille & 11,9 & 20,9 \\
\hline \multirow{4}{*}{ Taille du ménage } & $<8$ & 36,6 & 10,7 \\
\hline & {$[8-12]$} & 39,2 & 18,7 \\
\hline & [13-16] & 11,9 & 20,1 \\
\hline & $>16$ & 12,3 & 50,5 \\
\hline \multirow{5}{*}{ Classe d'âge } & [10-15] & 8,2 & 15,1 \\
\hline & [16-20] & 17,6 & 27,7 \\
\hline & [21-40] & 31,5 & 19,5 \\
\hline & [41-60] & 35,6 & 22,8 \\
\hline & $>60$ & 7,1 & 14,8 \\
\hline Niveau d'éducation & Aucun & 60,4 & 31,6 \\
\hline
\end{tabular}




\begin{tabular}{|c|c|c|c|}
\hline & Ecole coranique et autre $^{1}$ & 17,5 & 22,3 \\
\hline & Primaire & 16,8 & 25,0 \\
\hline & $\begin{array}{l}\text { Secondaire et post- } \\
\text { secondaire }\end{array}$ & 5,2 & 21,2 \\
\hline \multirow{3}{*}{ Groupe ethnique } & Peulh & 80,2 & 0 \\
\hline & Mossi & 6,7 & 99,7 \\
\hline & Autres ${ }^{2}$ & 13,1 & 0,3 \\
\hline \multirow{3}{*}{ Religion } & Chrétienne & 0,7 & 8,2 \\
\hline & Musulmane & 98,9 & 91,5 \\
\hline & Traditionnelle & 0,4 & 0,3 \\
\hline \multirow{3}{*}{ Statut matrimonial } & Marié & 76,9 & 53,6 \\
\hline & Célibataire & 23,1 & 45,6 \\
\hline & Autre $^{3}$ & & 0,8 \\
\hline \multirow{4}{*}{ Activité primaire } & Agriculture & 14,9 & 48,6 \\
\hline & Elevage & 10,8 & 0,3 \\
\hline & Agriculture et élevage & 72,4 & 41,2 \\
\hline & Autres $^{4}$ & 1,9 & 9,9 \\
\hline
\end{tabular}

${ }^{1}$ : école rurale, alphabétisation $;^{2}:$ Djerma, Haoussa et Gourounsi.

${ }^{3}$ : veuf $;{ }^{4}$ : ménagère, orpaillage, commerce, élève.

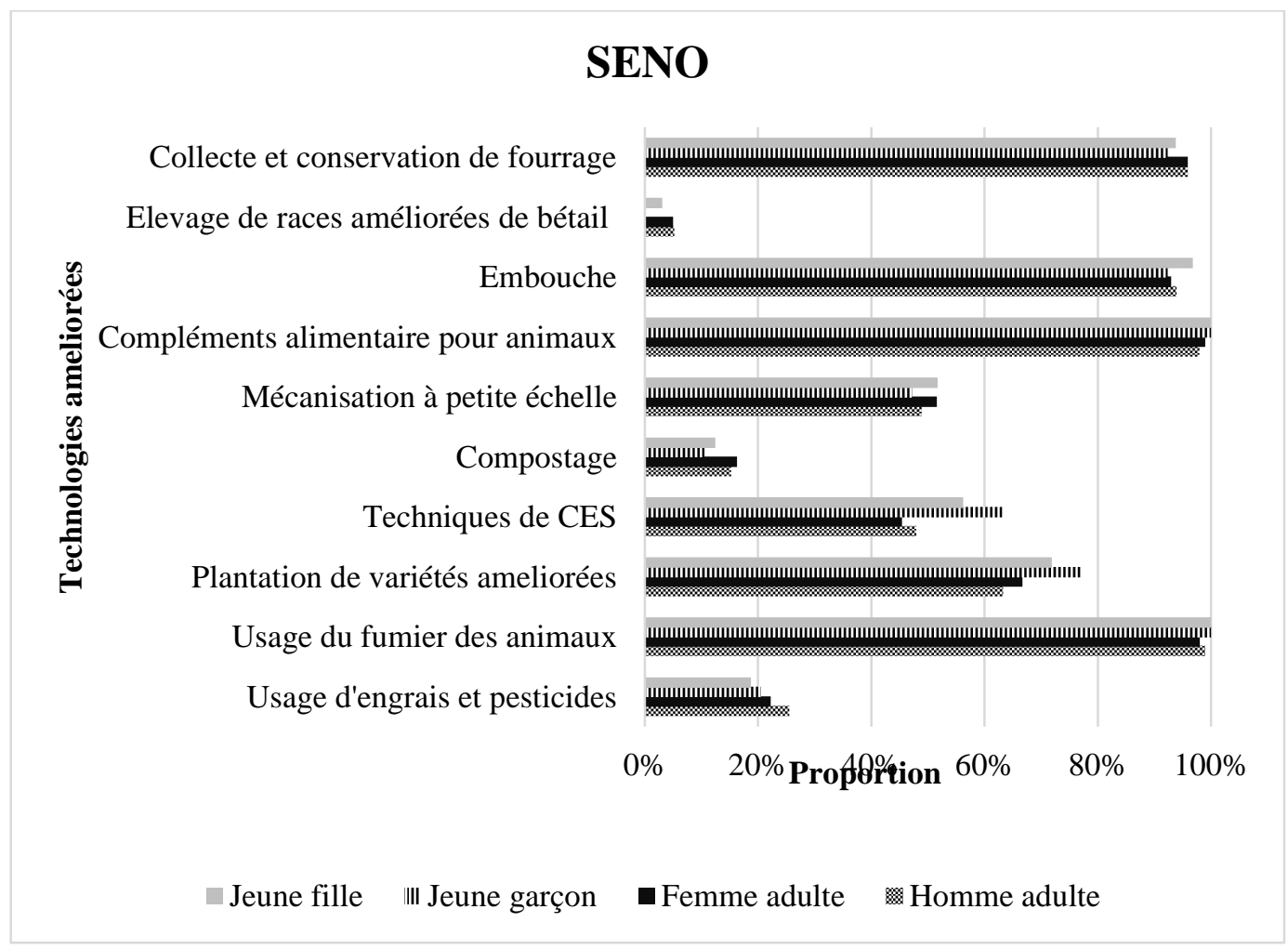

Figure 2 : Proportion de pratique des techniques d'intensification de la production agropastorale selon le genre au Séno. 
Tableau 2: Effet des caractéristiques sociodémographiques sur la pratique des technologies d'intensification dans la province du Séno.

\begin{tabular}{|c|c|c|c|c|c|c|c|}
\hline & $\begin{array}{l}\text { Usage de l'engrais } \\
\text { et des pesticides }\end{array}$ & $\begin{array}{l}\text { Usage du } \\
\text { fumier des } \\
\text { animaux }\end{array}$ & $\begin{array}{l}\text { Plantation de } \\
\text { variétés } \\
\text { améliorées }\end{array}$ & CES $^{*}$ & $\begin{array}{l}\text { Elevage de races } \\
\text { de bétail } \\
\text { améliorées }\end{array}$ & $\begin{array}{l}\text { Collecte et } \\
\text { conservation du } \\
\text { fourrage }\end{array}$ & $\begin{array}{l}\text { Mécanisation à } \\
\text { petite échelle }\end{array}$ \\
\hline \multicolumn{8}{|l|}{ Activité primaire } \\
\hline \multicolumn{8}{|l|}{ Agriculture } \\
\hline $\begin{array}{l}\text { Agriculture et } \\
\text { élevage }\end{array}$ & $0,32 * *$ & & $0,31 *$ & $0,20 * * *$ & & & $0,16 * * *$ \\
\hline \multicolumn{8}{|l|}{ Niveau d'éducation } \\
\hline \multicolumn{8}{|l|}{ Aucun } \\
\hline $\begin{array}{l}\text { Ecole coranique et } \\
\text { autre }\end{array}$ & & & $3,59 * * *$ & $5,76 * * *$ & & & $2,24 *$ \\
\hline Primaire & & $0,03 *$ & $2,82 *$ & $2,20^{*}$ & & & \\
\hline $\begin{array}{l}\text { Secondaire et post } \\
\text { secondaire }\end{array}$ & & & $13,73 *$ & $4,62 *$ & & & $4,11^{*}$ \\
\hline \multicolumn{8}{|l|}{ Taille de ménage } \\
\hline \multicolumn{8}{|l|}{$<8$} \\
\hline [8-12] & & & $0,41^{*}$ & & & & $0,54 *$ \\
\hline [13-16] & $2,58 *$ & & $0,12 * *$ & & & & \\
\hline$>16$ & $5,21 * *$ & & & & $7,91 *$ & & $2,91 *$ \\
\hline \multicolumn{8}{|l|}{ Groupe ethnique } \\
\hline \multicolumn{8}{|l|}{ Peulh } \\
\hline Mossi & $4 * *$ & & & & & $0,11 * *$ & \\
\hline Autres & & & & & & & $3,15 *$ \\
\hline \multicolumn{8}{|l|}{ Statut matrimonial } \\
\hline Célibataire & & & & & & & $0,19 *$ \\
\hline
\end{tabular}


K. NAPON et al. /Int. J. Biol. Chem. Sci. 14(3): 800-815, 2020

\begin{tabular}{ll}
\hline Groupe de genre & \\
\hline Jeune garçon & \\
\hline Jeune fille & $11,83 *$ \\
\hline Significativité $* * *=1 \% ; * *=5 \%, *=10 \%$ & $\mathrm{n}=267$
\end{tabular}

Tableau 3: Effet des caractéristiques sociodémographiques sur la pratique des technologies d'intensification dans la province du Yatenga.

\begin{tabular}{|c|c|c|c|c|c|c|c|c|c|c|}
\hline & $\begin{array}{l}\text { Usage de } \\
\text { l'engrais et des } \\
\text { pesticides }\end{array}$ & $\begin{array}{l}\text { Usage du } \\
\text { fumier des } \\
\text { animaux }\end{array}$ & $\begin{array}{l}\text { Plantation } \\
\text { de variétés } \\
\text { améliorées }\end{array}$ & CES & $\begin{array}{l}\text { Elevage } \\
\text { de races } \\
\text { de bétail } \\
\text { amélioré }\end{array}$ & $\begin{array}{l}\text { Usage de } \\
\text { compléments } \\
\text { alimentaire } \\
\text { pour animaux }\end{array}$ & Embouche & $\begin{array}{l}\text { Collecte et } \\
\text { conservation } \\
\text { du fourrage }\end{array}$ & Compostage & $\begin{array}{l}\text { Mécanisation à } \\
\text { petite échelle }\end{array}$ \\
\hline \multicolumn{11}{|l|}{$\begin{array}{l}\text { Activité } \\
\text { primaire }\end{array}$} \\
\hline $\begin{array}{l}\text { Agriculture } \\
\text { et élevage }\end{array}$ & $7,21 * *$ & & $1,75^{*}$ & & & $2,97 *$ & & $19,3 * *$ & $2,49 * *$ & \\
\hline Autres & & & & & $5,63 * * *$ & & $3,35 *$ & & $13,28 *$ & \\
\hline \multicolumn{11}{|l|}{$\begin{array}{l}\text { Niveau } \\
\text { d'éducation }\end{array}$} \\
\hline $\begin{array}{l}\text { Ecole } \\
\text { coranique et } \\
\text { autre }\end{array}$ & & & & & & $5,41 *$ & $3,81 * *$ & & & $4,86 *$ \\
\hline Primaire & & & $4,33 * *$ & & & $3,99 *$ & $2,81 *$ & & & \\
\hline $\begin{array}{l}\text { Secondaire } \\
\text { et post } \\
\text { secondaire }\end{array}$ & & & $4,32 * *$ & & & & & & & \\
\hline $\begin{array}{l}\text { Taille de } \\
\text { ménage }\end{array}$ & & & & & & & & & & \\
\hline
\end{tabular}


K. NAPON et al. /Int. J. Biol. Chem. Sci. 14(3): 800-815, 2020

\begin{tabular}{|c|c|c|c|c|c|c|}
\hline [8-12] & & & $0,19 *$ & & $3,08^{*}$ & \\
\hline [13-16] & $11,69 *$ & $0,37 *$ & $4,58 * *$ & $0,24 *$ & & \\
\hline$>16$ & $135,55^{* *}$ & & $2,48^{*}$ & $0,19 *$ & $2,14^{*}$ & $2,92 *$ \\
\hline \multicolumn{7}{|l|}{$\begin{array}{l}\text { Classe } \\
\text { d'age }\end{array}$} \\
\hline [16-20] & $2,86^{*}$ & & $2,02 *$ & $3,22 *$ & & $2,77 *$ \\
\hline [21-40] & $36,44 *$ & & $8,43^{* *}$ & & & $19,92 *$ \\
\hline$[41-60]$ & $103,64 * *$ & & $4,4^{*}$ & & & \\
\hline$>60$ & $28,89 *$ & & & & & \\
\hline \multicolumn{7}{|l|}{$\begin{array}{l}\text { Statut } \\
\text { matrimonial }\end{array}$} \\
\hline Célibataire & $0,09 *$ & & $0,13 * *$ & & $0,05^{*}$ & \\
\hline \multicolumn{7}{|l|}{$\begin{array}{l}\text { Groupe de } \\
\text { genre }\end{array}$} \\
\hline $\begin{array}{l}\text { Jeune } \\
\text { garçon }\end{array}$ & $208,42 * *$ & & $6,36^{*}$ & & & \\
\hline Jeune fille & $341,12 * *$ & & & & & \\
\hline
\end{tabular}




\section{YATENGA}

Collecte et conservation de fourrage

Elevage de races améliorées de bétail

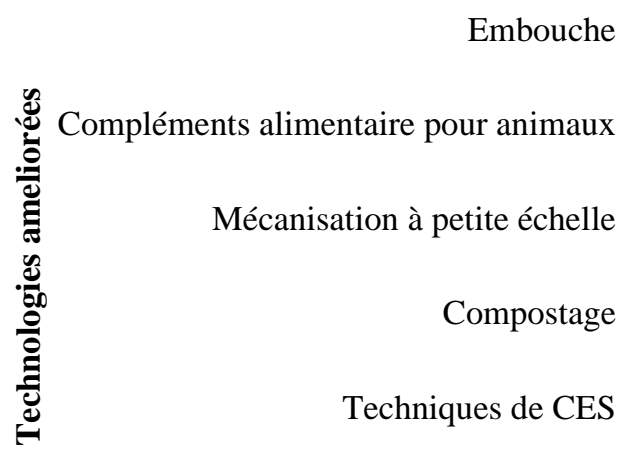

Plantation de variétés ameliorées

Usage du fumier des animaux

Usage d'engrais et pesticides

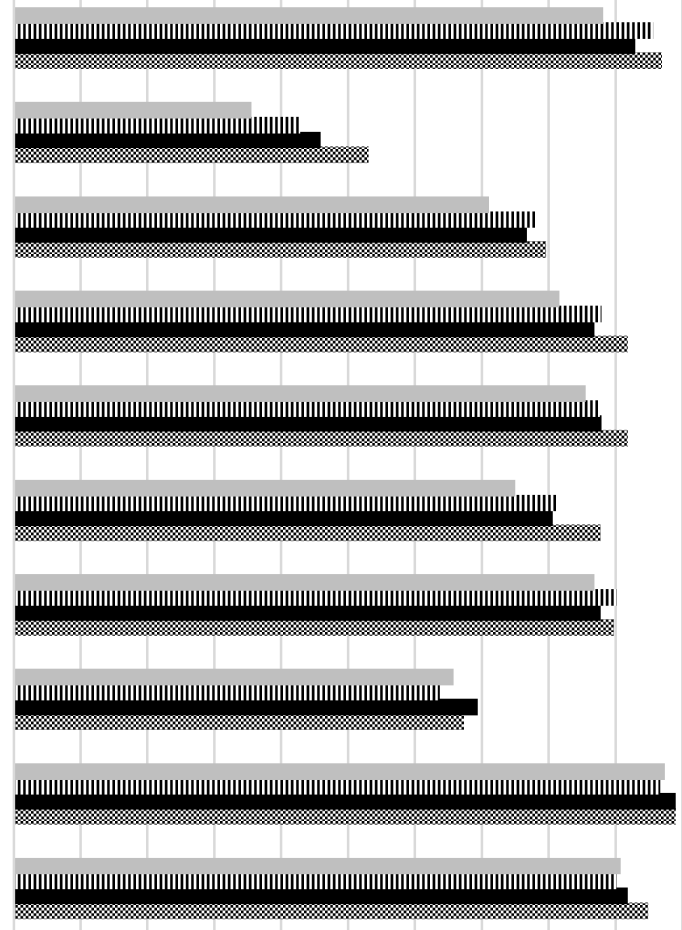

0\% 10\% 20\% 30\% 40\% 50\% 60\% 70\% 80\% 90\% $100 \%$

\section{Proportion}

$\square$ Jeune fille III Jeune garçon $\quad$ Femme adulte $\quad$ Homme adulte

Figure 3: Proportion de pratique des techniques d'intensification de la production agropastorale selon le genre au Yatenga.

\section{DISCUSSION}

Les technologies d'intensification durable de la production agropastorale prises en compte dans cette étude sont: l'usage de l'engrais et des pesticides, l'usage de la fumure animale, la plantation de variétés améliorées, les techniques de conservation des eaux et des sols, le compostage, la mécanisation à petite échelle pour la production végétale ; l'élevage de races de bétail améliorées, l'usage de compléments alimentaires pour animaux, l'embouche, la collecte et la conservation du fourrage pour la production animale.

\section{Différences dans l'utilisation des technologies d'intensification}

Il y'a une différence entre les provinces quant à l'adoption des technologies améliorées. Le niveau d'adoption des technologies améliorées est très élevé chez les répondants de la province du Yatenga par rapport à ceux de la province du Séno (Figures 4 et 5). Des quatre technologies d'intensification de la production animale considérées dans cette étude, l'élevage des races améliorées de bétail est le moins pratiqué par les répondants du Séno. Pour ce qui concerne les technologies d'intensification de la production végétales, les moins pratiquées sont le compostage, l'usage de 
l'engrais et des pesticides. Par contre, dans la province du Yatenga, les techniques d'intensification de la production animale et végétale sont bien pratiquées selon les répondants.

Cette situation peut s'expliquer par le fait que la province du Yatenga a une longue tradition dans la pratique des technologies améliorées. Elle a été depuis les années 80 le site d'expérimentation et de promotion des techniques de restaurations des sols, notamment les techniques de conservation des eaux et des sols par plusieurs projets et programmes (Botoni et Reij, 2009). Toute chose qui contribue à de meilleurs rendements agricoles (Ngenwi et al., 2011). Pendant ce temps, la province du Séno était une zone exclusivement pastorale jusqu'aux sècheresses des années 70. C'est cette situation qui aurait conduit les pasteurs à intégrer l'agriculture dans leurs activités et à produire de plus en plus de céréales pour leur propre consommation.

Selon les résultats, il n'y a pas de variation significative entre les groupes de genre de la proportion des répondants dans l'utilisation des techniques d'intensification suivantes : la collecte et la conservation du fourrage, l'utilisation des compléments alimentaires pour les animaux, l'utilisation des engrais et pesticides, de la fumure organique, des semences améliorées. Le niveau d'utilisation de l'engrais et de la fumure organique est le même pour les hommes, les femmes et les jeunes. Car plus de $90 \%$ des hommes, des femmes et des jeunes utilisent la fumure organique dans la province Séno, une zone fortement pastorale. Tandis que dans le Yatenga (zone agricole principalement) c'est plus de $90 \%$ qui utilisent les engrais et les pesticides. Pourtant, les travaux de Yabi et al. (2016) et de Dabiré et al. (2018) exposent que les femmes à titre personnel ont moins accès à l'engrais et à la fumure organique que les hommes. Les femmes mariées y ont accès grâce à leur mari (Dabiré et al., 2018). De plus, l'inaccessibilité des femmes aux équipements agricoles, constitue un frein à l'intensification de la production agricole étant entendu que c'est la force de travail la plus abondante qui est privée d'équipement de travail (Alwang et al., 2017).

Il y a cependant une variation non négligeable selon le genre de la proportion des répondants dans l'utilisation des races et semences améliorées. Les résultats montrent que, comparativement aux enquêtés masculins, les femmes et les filles ont un faible accès aux races et semences améliorées du fait de leur difficulté à obtenir un crédit agricole. Au Cameroun, Kumase et al. (2010) expliquent ce faible accès aux technologies améliorées des femmes par leur contact limité avec les services de vulgarisation. Pour Dabiré et al. (2018), bien que le taux d'utilisation des variétés améliorées (exemple : le sorgho) ne diffère pas entre les hommes et les femmes au Burkina Faso, il ressort néanmoins que les femmes ont moins accès aux semences améliorées que les hommes. Le faible accès, voire l'inaccessibilité aux différentes techniques selon les individus enquêtés, est dû principalement à la non disponibilité de la technologie, au manque d'information et de formation sur la technologie notamment le manque de capacité technique pour appliquer la technologie et aussi et surtout au prix élevé de certains intrants (engrais, pesticides, compléments alimentaires, etc.) nécessaires à l'application de la technologie. Sale et al. (2014) au Kenya ont trouvé que le faible usage des engrais comme alternative à la baisse de la fertilité des sols est dû à leur coût élevé et au manque de moyens des agriculteurs pour s'en procurer. De même, pour Sigué et al. (2018) la formation sur les techniques agricoles favorise l'adoption des technologies.

\section{Déterminants sociodémographiques de l'utilisation d'intensification des technologies}

Les facteurs déterminant l'adoption des innovations en milieu rural sont divers et dépendent de la technologie mais aussi des caractéristiques sociodémographiques du producteur. Dans cette étude nous avons trouvé que le niveau d'éducation, l'activité primaire, la taille du ménage, l'âge, le groupe genre et le groupe ethnique des individus sont les facteurs 
significativement associés à l'adoption des technologies. L'élevage des races améliorées est très peu pratiqué dans le Séno du fait de la faible résistance de ces races faces aux intempéries du sahel. Ce qui limite leur survie comparativement aux races locales. Le manque de ressources financières est aussi évoqué comme frein à l'acquisition des races améliorées. Yabi et al. (2016) trouvent que l'éducation est le facteur influant sur l'adoption d'une innovation technologique, car le producteur instruit est plus ouvert et apprécie facilement les nouvelles technologies.

La grande taille du ménage est positivement associée à l'adoption des variétés de céréales et de légumineuses améliorées. Ce résultat corrobore celui de Zakari (2017) qui a trouvé que la taille du ménage, la formation et le crédit agricole influencent positivement la probabilité d'adoption des variétés améliorées par les producteurs. Egalement, la taille du ménage est associée à l'utilisation de l'engrais, ce qui est différent des résultats de Yabi et al. (2016) pour lesquels, le sexe et l'appartenance à un groupement influencent positivement l'adoption de l'engrais ; de Zakari (2017) pour qui la formation agricole et le nombre d'animaux de trait sont des facteurs qui influencent positivement l'adoption de l'application des engrais chimiques. Sigué et al. (2018) au Burkina Faso trouvent que plus le nombre d'actifs agricoles est élevé et disponibles, plus les producteurs sont enclins à pratiquer la technique de la microdose. L'usage des compléments alimentaires pour les animaux est significativement associé (10\%) à l'activité primaire. Ce résultat est similaire à celui de Montcho et al. (2018) au Benin où $84 \%$ des éleveurs ont adopté les compléments alimentaires. L'usage des techniques de conservation des eaux et des sols est significativement associé à la taille du ménage, à l'activité primaire (notamment l'agropastoralisme) et au niveau d'éducation. Ce résultat est semblable à celui de Ndiritu et al. (2014) et de Zakari (2017) qui ont trouvé respectivement que les familles nombreuses, la formation et la possession d'animaux sont positivement associées à l'adoption des CES.
Pour Yabi et al. (2016), le sexe et l'âge du producteur jouent négativement sur la pratique de la lutte antiérosive en tant que technique de conservation des sols. Ce qui signifie que les femmes utilisent moins la lutte antiérosive que les hommes. De même, les jeunes sont plus disposés à l'utilisation de la pratique de lutte antiérosive comme stratégie de conservation des sols que les personnes âgées, parce que cette technique demande un effort physique. En cherchant donc à comprendre le rôle que les femmes peuvent jouer dans le processus d'adoption des technologies améliorées, Tene et al. (2013) ont trouvés que l'activité de transformation artisanale des produits agricoles est généralement réservée aux femmes. La pratique du compostage est significativement associée à l'activité primaire du répondant et à la taille du ménage. Jaza (2015) au Cameroun a trouvé que les agriculteurs issus de ménages de grande taille sont plus enclins à l'usage du compost que ceux issus de ménages de petite taille.

\section{Conclusion}

La présente étude se proposait d'identifier les différentes techniques d'intensification de la production agropastorale pratiquées selon le genre et de chercher les déterminants de la pratique de ces techniques par les populations. L'étude a révélé que les caractéristiques sociodémographiques influencent l'adoption des techniques d'intensification de la production agropastorale. Le niveau d'éducation, l'activité primaire, la taille du ménage, l'âge, le groupe genre et le groupe ethnique sont donc significativement associés à l'adoption des technologies d'intensification de la production agropastorale. Il est important de réduire les disparités qui existent entre les hommes, les femmes et les jeunes en rendant les ressources productives accessibles à tous sans distinction de genre. Ce qui va favoriser l'adoption des techniques d'intensification à tous les niveaux. Il est également important de mettre en œuvre des stratégies de vulgarisation et d'amélioration de la connaissance des producteurs sur la performance des différentes 
techniques pour leur totale appropriation dans les zones concernées. Toute chose qui va permettre d'améliorer durablement la productivité agricole.

\section{CONFLIT D'INTERETS}

Les auteurs déclarent qu'il n'y a aucun conflit d'intérêts concernant le manuscrit.

\section{CONTRIBUTIONS DES AUTEURS}

$\mathrm{KN}$ a mené le design des instruments d'enquête pour l'étude, a supervisé la collecte des données et a analysé et interprété les données puis préparé le manuscrit. AA a fait la revue du manuscrit. DECD a fait une révision critique du manuscrit. Tous les co-auteurs ont participé à la relecture du manuscrit et approuvé la version finale.

\section{REMERCIEMENTS}

Nous sommes reconnaissants aux répondants des sites de l'étude dans les provinces du Séno et du Yatenga au Burkina Faso pour leur participation dans cette étude. En outre, nous remercions les assistants de terrain pour la collecte et la saisie des données. Les auteurs sont seuls responsables des opinions exprimées dans ce manuscrit. Cette étude a été conduite dans le cadre du projet du Laboratoire d'intensification durable de Feed the Future financée par USAID (Agence de Développement Internationale des Etats-Unis).

\section{REFERENCES}

Alesina AF, Giuliano P, Nunn N. 2011. On the origins of gender roles: women and the plougth. National Bureau of Economic Research, no 17098. 45 p. https://doi.org/10.3386/w17098

Alwang J, Larochelle C, Barrera V. 2017. Farm Decision Making and Gender: Results from a Randomized Experiment in Ecuador. World Development, 92: 117129. DOI: https://doi.org/10.1016/j.worlddev.2016. 11.015 .

Botoni E, Reij C. 2009. La transformation silencieuse de l'environnement et des systèmes de production au Sahel:
Impacts des investissements publics et privés dans la gestion des ressources naturelles. 63 pages. http://portails.cilss.bf/IMG/pdf/Rapport_ Synthese_Etude_Sahel_Final.pdf.

Camara A, Dieng A, Mergeai G. 2015. Amélioration des systèmes de production mixtes en Afrique soudano-sahélienne. Rôle de l'espèce Stylosanthes hamata (L.) Taub. (Synthèse bibliographique). Biotechnologie Agronomie Société Environnement, 19(3) : 281-289. DOI : https://popups.uliege.be:443/17804507/index.php?id=12191.

Capitant S. 2017. Les populations à l'assaut des mines : économie rurale, de la contestation minière au Burkina Faso. In Anthropologie Des Prédations Foncières. Entreprises Minières et Pouvoirs Locaux, Leclerc-Olive M (Ed). Edition des Archives Contemporaines: Paris (France).

Croppenstedt A, Goldstein M, Rosas N. 2013. Gender and agriculture: Inefficiencies, segregation, and low productivity traps. The World Bank Research Observer, 28: 79-109.

DOI: https://doi.org/10.1093/wbro/lks024

Dabire I, Smale M, Theriault V. 2018. Le rôle du genre dans l'intensification de la production du sorgho au Burkina Faso : Une étude de méthodes mixtes. MSU International Development Working Paper, no 159. 31 pages.

Dorin B. 2014. Dynamiques agricoles en Afrique subsaharienne : une perspective à 2050 des défis de la transformation structurelle Rapport pour la Fondation pour l'agriculture et la ruralité dans le monde (FARM), Paris. 52 pages. http://hal.cirad.fr/cirad-

01113012/document

Parks MH, Christie ME, Bagares I. 2014. Gender and conservation agriculture: constraints and opportunities in the Philippines. GeoJournal, 80 : 61-77. DOI : https://doi.org/10.1007/s10708014-9523-4 
Herrera R, Ilboudo L. 2012. Les défis de l'agriculture paysanne : le cas du Burkina Faso. Cairn. Info L'Harmattan L'Homme et la Société, $1 \mathrm{n}^{\circ}$ 183-184, 83-95. https://www.cairn.info/revue-1-hommeet-la-societe-2012-1-page-83.htm

Hillenkamp I. 2011. Genre, systèmes agraires et vulnérabilités alimentaires une analyse nécessaire. Document de travail du Programme Genre, globalisation et changements, 4/2011. Genève. 53 pages.

Howard PL, Nabanoga G. 2007. Are There Customary Rights to Plants? An Inquiry Among the Baganda (Uganda), with Special Attention to Gender. World Development, 35: 1542-1563. DOI: https://doi.org/10.1016/j.worlddev.2006. 05.021

INSD. 2018a. Annuaire statistique 2017 de la région du Sahel. Direction régionale de l'institut national de la statistique et de la démographie du Sahel. 258 pages. http://cns.bf/IMG/pdf/annuaire_2017_du _nord.pdf

INSD. 2018b. Annuaire statistique 2017 de la région du Nord. Direction régionale de l'institut national de la statistique et de la démographie du Sahel. 211 pages. http://cns.bf/IMG/pdf/annuaire_2017_du _nord.pdf

Jaza FAJ. 2015. The determinants for the adoption of compost from household waste for crop production by farmers living nearby Yaoundé, Cameroon: descriptive and logit model approaches of analysis. International Journal of Biological and Chemical Sciences, 9(1): 308-328.

DOI: http://dx.doi.org/10.4314/ijbcs.v9i1.28

Kumase WN, Bisseleua H, Klasen S. 2010. Opportunities and constraints in agriculture: A gendered analysis of cocoa production in Southern Cameroon. Courant Research Centre: Poverty, Equity and Growth - Discussion Papers, no. 27, Courant Research Centre Poverty, Equity and Growth, Göttingen. 24 p. http://hdl.handle.net/10419/90510
Masuy-Stroobant G, Costa R, Baudewyns P. 2013. Analyser les Données en Sciences Sociales de la Préparation des Données à l'Analyse Multivariée. P.I.E Peter Lang: Bruxelles.

Montcho $\mathrm{M}$, Babatounde $\mathrm{S}$, Aboh AB, Houndonougbo F, Chrysostome AMC. 2018. Perception et adoption des innovations techniques en alimentation des ruminants au Benin. Agronomie Africaine, $\quad 30(1): \quad 31-45$. https://www.researchgate.net/publication /326493218

Ndiritu SW, Kassie M, Shiferaw B. 2014. Are there systematic gender differences in the adoption of sustainable agricultural intensification practices? Evidence from Kenya. Food Policy, 49: 117-127. DOI: https://doi.org/10.1016/j.foodpol.2014.0 6.010

Ngenwi AA, Tabi FO, Mafeni JM, Etchu KA. 2011. Actes du colloque 5. In Quelle Agriculture Pour Un Développement Durable de l'Afrique? Thiombiano TKS, Dialla E (Eds). CEDRES : Ouagadougou ; 797-805.

Peterman A, Behrman JA, Quisumbing AR. 2010. A Review of Empirical Evidence on Gender Differences in Non-land Agricultural Inputs. Technology, and Services in Developing Countries. ESA Working Paper, no. 11-11. https://doi.org/10.1007/978-94-0178616-4

Petit M. 2011. Pour une Agriculture Mondiale Productive et Durable. Editions Q. ed.

Quisumbing AR, Meinzen-dick R, Raney TL, Croppenstedt A, Behrman JA, Peterman A. 2014. Closing the Knowledge Gap on Gender in Agriculture. In Gender in Agriculture. Springer : Dordrecht ; 3-27. DOI: https://doi.org/10.1007/978-94017-8616-4

Sale A, Folefack DP, Obwoyere GO, Lenah Wati N, Lendzemo WV, Wakponou A. 2014. Changements climatiques et déterminants d'adoption de la fumure organique dans la région semi-aride de Kibwezi au Kenya. International Journal 
of Biological and Chemical Sciences, 8(2): 680-694. DOI: http://dx.doi.org/10.4314/ijbcs.v8i2.24

Sigué A, Labiyi IA, Yabi JA, Biaou G. 2018. Facteurs d'adoption de la technologie "Microdose" dans les zones agroécologiques au Burkina Faso. International Journal of Biological and Chemical Sciences, 12(5): 2030-2043. DOI: https://dx.doi.org/10.4314/ijbcs.v12i5.6

Smale M, Kergna A, Assima A. 2016. Genre, génération et intensification agricole : le cas de deux céréales de la savane soudanienne malienne. Feed the Future Innovation Lab for Food Security Policy Document de Travail, no 26. 36 pages.

Tene GLM, Havard M, Temple L. 2013. Déterminants socio-économiques et institutionnels de l'adoption d'innovations techniques concernant la production de maïs à l'ouest du Cameroun. Tropicultura, 31(2): 137-142. DOI :

http://www.tropicultura.org/text/v31n2/1 37.pdf

Theriault V, Smale M, Haider H. 2017. How Does Gender Affect Sustainable Intensification of Cereal Production in the West African Sahel? Evidence from Burkina Faso. World Development, 92: 177-191.

DOI: https://doi.org/10.1016/j.worlddev.2016. 12.003

Panel de Montpellier. 2013. L'intensification Durable: Un nouveau paradigme pour l'agriculture durable Africaine. Un rapport du Panel de Montpellier. Agriculture for Impact. London, UK.

Valdivia C. 2001. Gender, livestock assets, resource management, and food security: Lessons from the SR-CRSP. Agriculture and Human Values, 18: 27-39.

Wooldridge JM. 2009. Introductory Econometrics: a Modern Approach. McGraw-Hill: New York, USA.

Yabi JA, Bachabi FX, Labiyi IA, Ode CA, Ayena RL. 2016. Déterminants socioéconomiques de l'adoption des pratiques culturales de gestion de la fertilité des sols utilisées dans la commune de Ouaké au Nord- Ouest du Bénin. International Journal of Biological and Chemical Sciences, 10(2): 779-792. DOI: https://doi.org/http://dx.doi.org/10.4314/i jbcs.v10i2.27

Zakari S. 2017. Adoption des technologies et pratiques d'agriculture intelligente face au climat dans les sites CCAFS (Niger). Rapport technique. 47 pages. https://doi.org/10.13140/RG.2.2.25400.7 5526 . 